Swarthmore College

Works

3-1-2006

\title{
Path Integral Monte Carlo Simulations Of Positronium Annihilation: From Micropores To Mesopores
}

Amy Lisa Graves

Swarthmore College, abug1@swarthmore.edu

P. A. Sterne

Follow this and additional works at: https://works.swarthmore.edu/fac-physics

Part of the Physics Commons

Let us know how access to these works benefits you

\section{Recommended Citation}

Amy Lisa Graves and P. A. Sterne. (2006). "Path Integral Monte Carlo Simulations Of Positronium Annihilation: From Micropores To Mesopores". Physical Review B. Volume 73, Issue 9. D0I: 10.1103/ PhysRevB.73.094106

https://works.swarthmore.edu/fac-physics/95

This work is brought to you for free by Swarthmore College Libraries' Works. It has been accepted for inclusion in Physics \& Astronomy Faculty Works by an authorized administrator of Works. For more information, please contact myworks@swarthmore.edu. 


\title{
Path integral Monte Carlo simulations of positronium annihilation: From micropores to mesopores
}

\author{
Amy L. R. Bug* \\ Department of Physics and Astronomy, Swarthmore College, Swarthmore, Pennsylvania 19081, USA \\ P. A. Sterne \\ Lawrence Livermore National Laboratory, Livermore, California 94550, USA \\ (Received 23 August 2005; revised manuscript received 23 November 2005; published 8 March 2006)
}

\begin{abstract}
Path integral Monte Carlo (PIMC) can reproduce the results of simple analytical calculations in which a single quantum particle is used to represent positronium within an idealized spherical pore. Our calculations improve on this approach by explicitly treating the positronium as a two-particle $e^{-}, e^{+}$system interacting via the Coulomb interaction. We study the lifetime and the internal contact density, $\kappa$, which controls the selfannihilation behavior for positronium in model spherical pores as a function of temperature and pore size. We compare the results with both PIMC and analytical calculations for a single-particle model.
\end{abstract}

DOI: 10.1103/PhysRevB.73.094106

\section{INTRODUCTION}

Positron annihilation lifetime spectroscopy (PALS), and angular correlation of annihilation radiation (ACAR), which measures the momentum distribution of annihilating electrons, are used extensively in order to determine the nature of voids and defects in insulating materials. ${ }^{1}$ Lifetime spectra contain signals associated with positrons annihilating from various states in the system, such as free positrons, positrons trapped at defects, and positrons in the form of positronium, a hydrogenlike bound electron-positron state. The signal from positrons annihilating in the spin-triplet orthopositronium (o-Ps) state is of particular interest for applications involving porous materials. ${ }^{2}$ The self-annihilation rate of $o$-Ps in a vacuum is $\Gamma_{0}=(142 \mathrm{~ns})^{-1}$, but this rate is increased by a "pick-off" process in which the positron annihilates with an electron in the surrounding material, resulting in a much shorter lifetime. ${ }^{3}$ Since the probability of pick-off annihilation depends on the size of the open volume region containing the positronium, the lifetime of the $o$-Ps state can be used as a sensitive probe of the size of open volume defects in materials. Of course, analytical or computational theory is needed in order to convert a lifetime or momentum spectrum into an estimation of the free volume fraction or distribution of pore spaces. ${ }^{4}$ Calculations which can provide simplified rules for dealing with experimental data sets are desirable. For example, while everyone realizes that voids in polymeric solids, zeolites, and so on are not isolated, spherical pores, the Tao-Eldrup model, ${ }^{5}$ which is based on this idea continues to be an extremely popular way to understand void sizes in these systems. ${ }^{6}$ Corrections to the Tao-Eldrup prediction, say for nonspherical voids and/or soft walls, ${ }^{7-10}$ are certainly useful. Yet a simple and general prediction of pore sizes from lifetime data remains elusive. ${ }^{11}$

Here, we discuss Ps within idealized spherical pores, although it is straightforward with this method to study Ps solvated in realistic liquids and embedded in solid matrices. $^{12,13}$ Path integral Monte Carlo (PIMC) has been used, for example, to investigate self-trapped Ps states in fluids, ${ }^{14}$ and to find free volume within a polymer. ${ }^{15}$ While
PACS number(s): 78.70.Bj, 71.60.+z

the model of interest in this paper corresponds only loosely to a real system, the inherent improved physical description offers insight into Ps behavior in real systems. Our treatment also enables one to assess the reliability and range of the applicability of existing, less fundamental models of Ps. In contrast to most previous computational and analytical work, we treat Ps as a two-particle electron-positron state bound by the Coulomb interaction. This allows us to assess the accuracy of existing methods that treat Ps as a single quantum particle, and to look at experimental observables that depend explicitly on the two particle nature of Ps. A refinement of our earlier procedure, ${ }^{16}$ the use of the exact Coulomb propagator described in the section below, allows us to determine the internal contact density, $\kappa$, which controls the rate of self-annihilation of Ps.

\section{METHODS AND THEORY}

PIMC is a method for sampling from the canonical density matrix, $\hat{\rho}(\beta)=\exp (-\beta \hat{H})$, for systems of light particles. It thus allows the calculation of thermal averages of observables; $\langle\hat{A}\rangle=\operatorname{Tr} \hat{\rho} \hat{A} / Q$, where $Q \equiv \operatorname{Tr} \hat{\rho}$. Here, $\hat{H}$ is the Hamiltonian, $\beta$ the inverse temperature, and $\hat{A}$ is an observable of interest. PIMC methods for simulating light particles are described in detail in several excellent reviews. ${ }^{17}$ In this calculation, $e^{+}$and $e^{-}$are represented as polymeric chains of entities known as "beads." The density of the positron beads at location $\mathbf{r}^{+}$is the quantity of interest, and this is constructed by sampling the thermal density,

$$
\begin{aligned}
\rho(\mathbf{R}, \mathbf{R} ; \beta) \equiv & \langle\mathbf{R}|\hat{\rho}(\beta)| \mathbf{R}\rangle \\
\approx & \int d \mathbf{R}_{1} \cdots d \mathbf{R}_{P-1} \prod_{i=1}^{P} K_{0}\left(\mathbf{R}_{i-1}, \mathbf{R}_{i} ; \epsilon\right) \\
& \times K_{C}\left(\mathbf{R}_{i-1}, \mathbf{R}_{i} ; \epsilon\right) K_{e x t}\left(\mathbf{R}_{i-1}, \mathbf{R}_{i} ; \epsilon\right),
\end{aligned}
$$

where $\mathbf{R}$ represents the six coordinate variables $\left(\mathbf{r}^{+}, \mathbf{r}^{-}\right) . P$ is a discretization variable, and $\epsilon \equiv \beta / P$. Equation (1) is exact in the limit that $P \rightarrow \infty$. Above, $\mathbf{R}_{P} \equiv \mathbf{R}_{0} \equiv \mathbf{R}$. 
$K_{0}$ is the free-particle (kinetic) density matrix,

$$
\begin{aligned}
K_{0}\left(\mathbf{R}, \mathbf{R}^{\prime} ; \epsilon\right)= & \left(\frac{m}{2 \pi \hbar^{2} \epsilon}\right)^{3} \\
& \times \exp \left(-\frac{m}{2 \hbar^{2} \epsilon}\left[\left(\mathbf{r}^{+}-\mathbf{r}^{\prime+}\right)^{2}+\left(\mathbf{r}^{-}-\mathbf{r}^{\prime-}\right)^{2}\right]\right) .
\end{aligned}
$$

The form of $K_{0}$ is like that of the partition function for two independent, classical, ring polymers with harmonic bonds. ${ }^{18}$ These two "polymers" are coupled by the additional terms in Eq. (1). $K_{C}$ is the part of the propagator that, when multiplied by $K_{0}$, produces the full Coulombic propagator for an isolated pair of charges. $K_{C}$ has been tabulated numerically by Pollock. ${ }^{19}$ This refinement, which replaces the Yukawa form used in our previous work, ${ }^{16,20}$ provides a more reliable and accurate approach for treating the Coulomb interaction between the electron and positron, and for calculating the internal contact density, $\kappa$. The term $K_{\text {ext }}\left(\mathbf{R}, \mathbf{R}^{\prime} ; \epsilon\right)$ in Eq. (1) arises from an external potential; in this case, one provided by the pore walls. For spherical pores with hard walls, we use either an effective form due to Kalos and Whitlock ${ }^{21}$ or, for better accuracy for the equivalent number of beads, a form derived by $\mathrm{Cao}$ and Berne from scattering theory. ${ }^{22}$ For soft walls which would exert a potential $V_{\text {ext }}\left(\mathbf{r}^{+}, \mathbf{r}^{-}\right)$, a local, "primitive" form can be used: $\rho_{\text {ext }}\left(\mathbf{R}_{\mathbf{i}-\mathbf{1}}, \mathbf{R}_{i} ; \epsilon\right)$ $\equiv \exp \left[-\epsilon V_{\text {ext }}\left(\mathbf{r}_{\mathbf{i}}^{+}, \mathbf{r}_{\mathbf{i}}^{-}\right)\right]$. In a real solid, correlation potentials will exist between the particles in Ps and the electrons and ions in the solid. An important effect of interactions with solid electrons is dielectric screening. These dielectric effects, which are not explicitly included here, are addressed elsewhere. ${ }^{23}$

The annihilation rate (inverse lifetime) of Ps in a solid can be written as ${ }^{24}$

$$
\Gamma=\kappa \Gamma_{0}+\Gamma_{\text {p.o. }},
$$

with the self-annihilation rate, $\kappa \Gamma_{0}$, and the pick-off annihilation rate, $\Gamma_{\text {p.o. }}$, operating in parallel. $\left(\Gamma_{0}\right.$ is the selfannihilation rate in a vacuum.) In order to facilitate comparison with other studies, a pick-off at a rate of $2 \mathrm{~ns}^{-1}$ (the spin-averaged rate of annihilation of Ps in a vacuum) is assumed to exist when a positron lies within a shell of thickness $\Delta$ on the pore surface, in which the electronic density is posited to reside. This model is, admittedly, an extreme oversimplification of a detailed calculation of quantum chemistry in which the overlap between positronic and calculated electronic densities determine the annihilation rate. It was fit to experiment by Nakanishi and Jean (see Ref. 5) with excellent results over several orders of magnitude of pore radii. In the ensuing years, it has become the "standard model" for this type of analysis. Thus, in order to understand the capabilities and limitations of the two-particle PIMC simulation, this standard model of pick-off behavior will be utilized.

Thus, the lifetime, $\tau$, will be calculated (in ns) as

$$
\tau^{-1} \equiv \Gamma=\kappa \Gamma_{0}+2 \int_{r=R_{c}-\Delta}^{r=R_{c}} n_{+}(\mathbf{r}) d^{3} r,
$$

where $n_{+}(\mathbf{r})$ is the positron density at location $\mathbf{r}$, and the pore radius is denoted as $R_{c}$. In order to compare with a single- particle model of Ps within spherical pores, we assign thermal weights and sum the appropriate spherical Bessel function contributions for a sphere of given size and temperature and calculate the single-particle probability distribution,

$$
\mathrm{P}_{S P I B}(r)=N \sum_{l, n}(2 l+1)\left[j_{l}\left(\alpha_{l, n} r / R_{c}\right)\right]^{2} r^{2} \exp \left(-\beta E_{l, n}\right),
$$

where $\alpha_{l n}$ is the $n$th zero of $j_{l}, E_{l, n}=\left(\alpha_{l, n}^{2} / 2 m R_{c}^{2}\right)$ in atomic units, and $N$ is a normalization. (The mass, $m$, will frequently be taken as $m=2$ when we compare results using $\mathrm{P}_{\text {SPIB }}$ with the two-particle PIMC calculation, in which $e^{+}$and $e^{-}$each have the mass $m=1$.) To find the Ps lifetime using this model, $\mathrm{P}_{S P I B}$ would be integrated over the region of width $\Delta$ in the manner of $n_{+}$in Eq. (4). ${ }^{25}$ This integration can be done analytically using the equality: ${ }^{26}$

$$
\begin{aligned}
\int_{R_{c}-\Delta}^{R_{c}} r^{2}\left(j_{l}(\lambda r)\right)^{2} d r= & \frac{\pi}{4 \lambda}\left[r ^ { 2 } \left\{\left(J_{l+1 / 2}(\lambda r)\right)^{2}\right.\right. \\
& \left.\left.-J_{l-1 / 2}(\lambda r) J_{l+3 / 2}(\lambda r)\right\}\right]_{R_{c}-\Delta}^{R_{c}} .
\end{aligned}
$$

A small amount of simplification arises from the fact that $J_{l+1 / 2}\left(\lambda R_{c}\right)=0$ in the case that $\lambda=\alpha_{l, n} / R_{c}$, in accord with the terms of Eq. (5). An analogous calculation was done previously for cubic voids. ${ }^{27}$

The internal contact density, $\kappa$, appearing in Eqs. (3) and (4) is the factor by which the square of the Ps orbital, $\phi\left(\mathbf{r}_{+}, \mathbf{r}_{-}\right)$, in a material differs from its vacuum value for coincident particles. Thus,

$$
\kappa=8 \pi a_{0}^{3} \int\left|\phi\left(\boldsymbol{r}_{+}, \boldsymbol{r}_{-}\right)\right|^{2} \delta\left(\boldsymbol{r}_{+}-\boldsymbol{r}_{-}\right) d^{3} \boldsymbol{r}_{+} d^{3} \boldsymbol{r}_{-} .
$$

Changes in $\kappa$ can alter the lifetime of the shorter-lived singlet state $p$-Ps; a high-resolution spectrometer is required to see this effect. Normally for $o$-Ps, the second term of Eq. (3) is dominant. However, an applied magnetic field ("magnetic quenching") allows one to deduce $\kappa$ in both PALS and ACAR experiments. Both dielectric screening and polarization should reduce $\kappa$ from unity. Indeed, experiments find values from $\kappa \approx 1$ (some silaceous zeolites, polymers, and noble fluids) to $\kappa<0.1$ (some ionic crystals). ${ }^{24}$ Interestingly, the prevalent spherical "particle-in-a-spherical-box" (PIB) model, ${ }^{5}$ widely used to interpret data on micropores in molecular solids, would result in an increase in $\kappa$ due to spherical confinement. ${ }^{28,29}$ Competition between the effects of compression (spherical or asymmetrical) and dilation of the Ps orbital, and their net effect on $\kappa$, are calculable with our PIMC method. Explicit interactions with solid electrons and ions will affect both $\kappa$ and the self-annihilation rate. In keeping with the approximation used for the potential, these effects will be ignored except to the extent that they are represented by the empirical choice of shell thickness, $\Delta$. In another work screening effects have been included by treating the pore as a cavity in a uniform dielectric material. ${ }^{23}$ Indeed, in that work, we find that bulk polarizability lowers $\kappa$, but in micropores, $\kappa$ remains greater than unity. Thus, realistically low values of $\kappa$ seem to require a detailed model of the microscopic electric field, produced by the response of molecules that define the pore or bubble. 
TABLE I. Calculated $o$-Ps lifetimes and contact densities in cavity of radius $R_{c}$. "SPIB" denotes a single particle-in-a-box model, with the radial density given by Eq. (5). For these groundstate data, this is equivalent to Eq. (8).

\begin{tabular}{lccc}
\hline \hline $\begin{array}{l}R_{c} \\
(\text { a.u. }\end{array}$ & $\begin{array}{c}\tau \\
(\mathrm{ns})\end{array}$ & $\begin{array}{c}\tau_{S P I B} \\
(\mathrm{~ns})\end{array}$ & $\kappa$ \\
\hline$\infty$ & & & 1.0 \\
12 & $7.8(2)$ & 4.7 & $1.02(2)$ \\
10 & $4.7(2)$ & 3.0 & $1.04(2)$ \\
8 & $2.4(1)$ & 1.7 & $1.10(5)$ \\
6 & $1.1(1)$ & 0.92 & $1.20(5)$ \\
5 & $0.73(3)$ & 0.68 & $1.45(5)$ \\
4.75 & $0.67(3)$ & 0.63 & $1.50(5)$ \\
\hline \hline
\end{tabular}

\section{RESULTS FOR MICROPORES:}

Table I shows the calculated lifetime $\tau \approx \Gamma_{\text {p.o. }}^{-1}$, for $o$-Ps in a small, spherical pore (infinite potential well). Lifetime is found from Eq. (4) with $\Delta=3.13$ a.u.. A temperature of 0.01 a.u. $(\beta=100)$ was chosen for swift convergence for pore radii $R_{c}<10.0$. This is sufficiently low that the system is approximately in its ground state. Values of $\beta=200,300$ were used for pores with $R_{c}=10.0,12.0$, respectively. Several million Monte Carlo (MC) passes (several thousand uncorrelated configurations) were used. Bead numbers $P=600$, 800 , and 1200 were used for $\beta=100,200$, and 300, respectively. The ratio $\mathrm{P} / \beta$ must be sufficiently large in order to correctly calculate the internal structure of Ps.

Single-particle PIMC simulations in micropores reproduce the analytical, ground-state single-PIB results precisely, verifying the accuracy of our implementation. Table I shows that the two-particle model gives smaller rates and/or longer lifetimes. ${ }^{16}$ Figure 1 plots the ground-state single-PIB result for the probability of $e^{+}$to lie within $\Delta$ of the pore wall: ${ }^{5}$

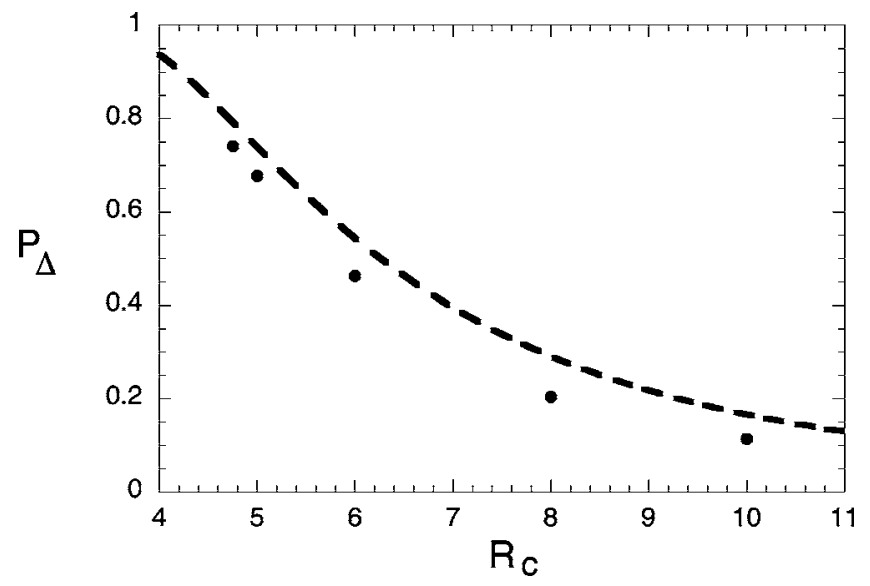

FIG. 1. Probability density, $P_{\Delta}$, for $e^{+}$to lie within a distance $\Delta=3.13$ a.u. of the cavity wall. Dashed line: Eq. (8); filled points: two-particle PIMC simulation.
TABLE II. Calculated values of electronic shell thickness, $\Delta$, in a.u. These are derived given $R_{c}$ and experimental lifetime. For the purpose of calculation, the latter are identified with the singleparticle lifetimes, $\tau_{S P I B}$ in the corresponding lines of Table I, since these are accepted as good estimates of true lifetimes in experimental systems.

\begin{tabular}{lc}
\hline \hline $\begin{array}{l}R_{c} \text { (a.u.) } \\
12\end{array}$ & \multicolumn{1}{c}{$\Delta$} \\
10 & $3.7(1)$ \\
6 & $3.6(1)$ \\
5 & $3.38(2)$ \\
\hline \hline & \\
& $\mathrm{P}_{\Delta}=\int_{r=R_{c}-\Delta}^{r=R_{c}} n_{+}(\mathbf{r}) d^{3} r$ \\
& $\equiv\left[1-\frac{R_{c}-\Delta}{R_{c}}+\frac{1}{2 \pi} \sin \left(\frac{2 \pi\left(R_{c}-\Delta\right)}{R_{c}}\right)\right]$.
\end{tabular}

Figure 1 also shows two-particle simulation data at a number of pore radii. The results indicate that single- and twoparticle models predict very different pore radii for a given lifetime value. For example, the two-particle simulation assigns a pore radius of $10.0 \mathrm{a}$.u. to a Ps experiment with a lifetime of 4.7 ns. However, Eq. (8) would predict that this lifetime corresponds to a radius which is fully $20 \%$ larger, 12.0 a.u. Mesopore calculations (below) show even more dramatic discrepancies between lifetimes predicted by the one- and two-particle models.

Another way to look at this disagreement is to consider the value $\Delta=3.13 \mathrm{a}$.u. which arises from a one-parameter fit of the Tao-Eldrup model to experimental annihilation data, both lifetime and ACAR, in solids. (A slightly larger value of $\Delta=3.5$ a. u. is fit to certain liquid-bubble systems, and is also said to be a better fit to certain systems with pores of extreme sizes. ${ }^{8}$ ) The fit presumes that cavity volumes are known by other means (e.g., porosimetry or crystal structure), that cavities are spherical, and the only free parameter of the model is the thickness of the electronic layer. Suppose that we attempted such a fit for the two-particle model. For each known value $R_{c}$, suppose we were told that the experiment yielded $\tau_{S P I B}\left(R_{c}\right)$ as listed in Table I. What thickness would we attribute to the electronic layer based on our two-particle calculation? (In other words, what value of $\Delta$ would make the calculated $\tau$ equal to the SPIB value?) We would derive the values seen in Table II. There is a systematic (with cavity size) variation seen in these predicted values. This is another way to gauge the disagreement between the one- and twoparticle models of Ps in a cavity.

Additionally, one might compare these results with the modification of the size of $\Delta$ necessary in order for Ref. 27 to reconcile rectangular with spherical pore model results. In that work, $\Delta$ was assigned a value of 3.40 a.u. for use in cubic pores. Table II suggests that here a revision in the electronic layer thickness of comparable size is necessary. Unlike the revision in Ref. 27 which worked for a large range of pore sizes, our revised $\Delta$ is a function of $R_{c}$ for 

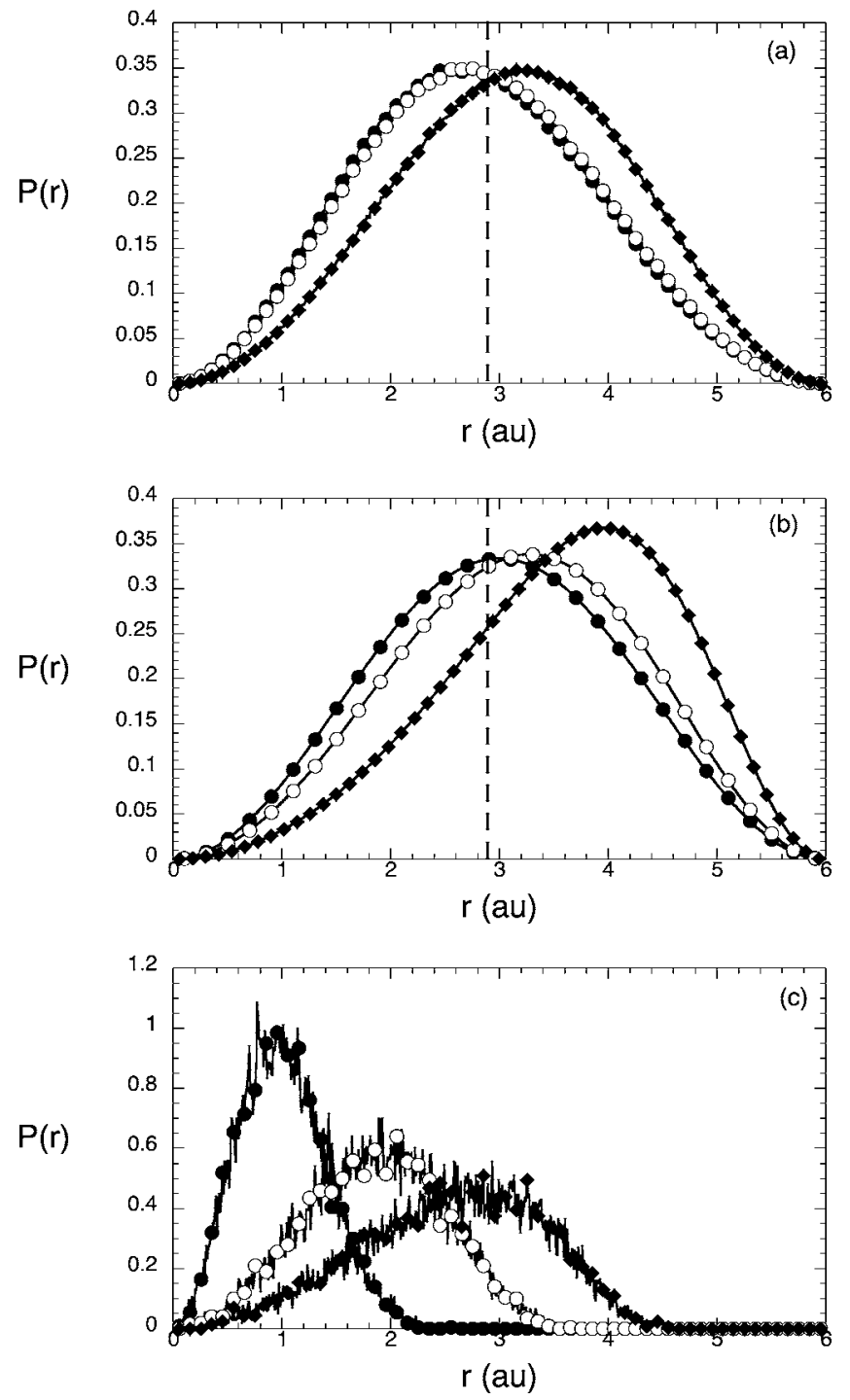

FIG. 2. Probability density, $P(\mathrm{r})$, within $R_{c}=6$ a.u. cavity. The data are represented by lines, with a subset of points labeled by symbols. Solid black circles; $\beta=100$, open circles; $\beta=30$, solid diamonds; $\beta=10$. Dashed vertical line indicates radial position located $\Delta$ from the pore wall. (a) $P(r)$ for $e^{+}$of Ps. (b) $\mathrm{P}_{S P I B}(\mathrm{r})$, according Eq. (5) with $m=2$. (c) $\mathrm{P}(\mathrm{r})$ for centroids of Ps chains.

small pores. Data on mesopores will be considered in the following section.

For a given spherical pore size, one expects the lifetime to decrease with temperature as higher angular momentum states, which have more weight near the pore wall, are occupied. For example, Fig. 2(b) shows the radial distribution function $\mathrm{P}_{S P I B}$ within an $R_{c}=6$ a.u. micropore for $\beta=10,30$, and 100 a.u.. Data from a single-chain PIMC simulation with $m=2$ and Eq. (5) are in excellent agreement with this figure, which includes all terms in Eq. (5) which contribute with a weight of at least $0.1 \%$ when compared with the leading term. (This amounts to, for $R_{c}=6$, summing ten states for $\beta=10$; and fewer for larger $\beta$, with only one state required for $\beta$ greater than approximately 50 a.u.) The distributions of $e^{+}$density for the two-particle model of Ps are shown in Fig. 2(a). Some differences are notable. Though temperature decreases lifetime, the effect is less pronounced for the two-

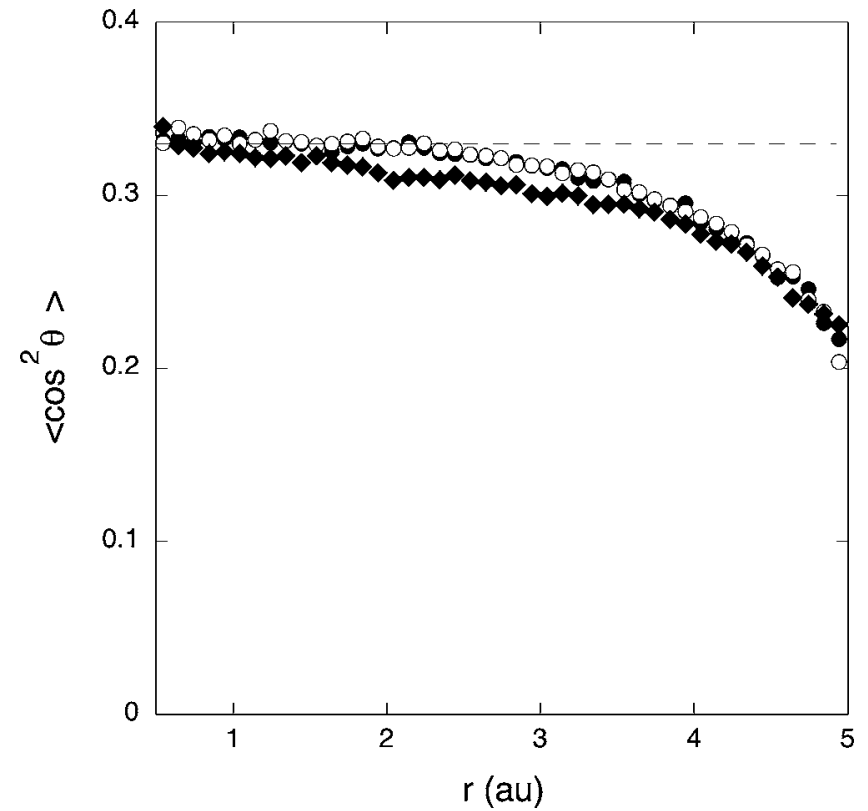

FIG. 3. Expectation value, $\left\langle\cos ^{2} \theta\right\rangle$. The angle $\theta$ is defined by the relative vector, $\mathbf{r}_{+-}$, and the vector pointing directly toward the pore wall. Here, $R_{c}=6$ a.u.. Solid circles: $\beta=100$; open circles: $\beta$ $=30$; solid diamonds: $\beta=10$. Dashed line indicates the expectation value for an isotropic distribution of orientations: $\left\langle\cos ^{2} \theta\right\rangle=\frac{1}{3}$.

particle calculation, since the two-chain distributions are less strongly weighted at larger radii than are spherical PIB (SPIB) distributions, leading to longer lifetimes: $0.84 \mathrm{~ns}$ (vs $0.66 \mathrm{~ns}$ for SPIB), 1.05 (vs 0.82) and 1.07 (vs 0.91) ns for $\beta=10,30$, and 100 , respectively. For $\beta=10$ and 30 the SPIB radial density seems to change more dramatically than does the density of the $e^{+}$of Ps. Nevertheless, the trend is clear. Interestingly, the centroid of the $e^{+}$and $e^{-}$chains has a distribution which moves out more noticeably in radius with temperature [Fig. 2(c) $].{ }^{30}$ Note that the centroid plot in Fig. 2(c) has much lower statistics than the positron density in Fig. 2(a) since each Ps chain has many beads contributing to the positron probability density, but only one centroid. The centroid is the PIMC degree of freedom most closely associated with a classical particle. Hence, in the high temperature and/or large cavity limit, we expect the centroid and the single-bead distributions to become more similar. The data of Fig. 2, however, embodies cases where the distributions are quite different. The $e^{+}$energy states are manifestly quantized, with a small number of low-lying states contributing.

The orientation of the Ps atom near a solid surface is likely to be important in determining the details of the pickoff lifetime. One might guess that the Ps atom would orient preferentially near a surface so as to minimize its free energy. In Fig. 3 one sees that in this hard, spherical cavity, the relative coordinate vector, $\mathbf{r}_{+-} \equiv \mathbf{r}_{+}-\mathbf{r}_{-}$is indeed more likely to be perpendicular to the cavity wall when the atom is in close proximity to the wall. In this figure, we plot $\left\langle\cos ^{2} \theta\right\rangle$, where $\theta$ is the angle between $\mathbf{r}_{+-}$and the radial direction, defined by the unit vector from the center-of-mass of a pair of Ps beads, $\left(\mathbf{r}_{+}+\mathbf{r}_{-}\right) / \mathbf{2}$, to the pore's center. In the small cavity of Fig. 3, it is only quite near the center that $\left\langle\cos ^{2} \theta\right\rangle$ takes on its isotropic value of $1 / 3$. It drops precipitously to 
zero in the region of interest, within $\Delta$ of the pore wall. (Data become very noisy at the end points, owing to the small number of observations of Ps at these locations.) This drop is seen in mesopores as well (Sec. IV). A perceptible dependence on temperature can be seen in Fig. 3; with the highest temperature, $\beta=10$, corresponding to more restriction of the orientation of Ps at the intermediate cavity radii. This is, perhaps, due to the expansion of the Ps orbital with temperature. The $e^{+}$and $e^{-}$have an expected separation which is roughly $10 \%$ larger at $\beta=10$ than at $\beta=30$ or 100 . (These expected separations are, nevertheless, all somewhat smaller than the free Ps ground-state value of 3 a.u.) This larger Ps atom is more poorly accommodated in a tiny pore, and the $e^{+}, e^{-}$pair is more strongly inhibited from orienting "end-on" to the pore wall.

PIMC allows a calculation of the internal state of Ps, hence the internal contact density, $\kappa$, as in Eq. (7). Although a hydrogen atom in the center of a hard spherical cavity can be solved exactly, ${ }^{28,29}$ the case of electron and positron wave functions vanishing on a sphere is a different problem that does not seem amenable to an analytic solution. In a small cavity, the $e^{+}$and $e^{-}$wave functions are compressed, resulting in a higher contact potential than in an unconstrained system, thereby increasing the self-annihilation rate. Table I shows the computed values of $\kappa\left(R_{c}\right)$, affirming the idea ${ }^{29}$ that only tiny pore radii will increase $\kappa$ significantly. Yet, for example, the confinement of a spherical bubble of radius $R_{c}=8$ a.u. (typical in a molecular liquid) increases $\kappa$ by $10 \%$. This is meaningful, given that the net experimental change tends to be a reduction of $20 \%$ or less in many liquids and solids of interest. ${ }^{24,9}$ As mentioned previously, calculation of a realistic, reduced $\kappa$ appears to require the kind

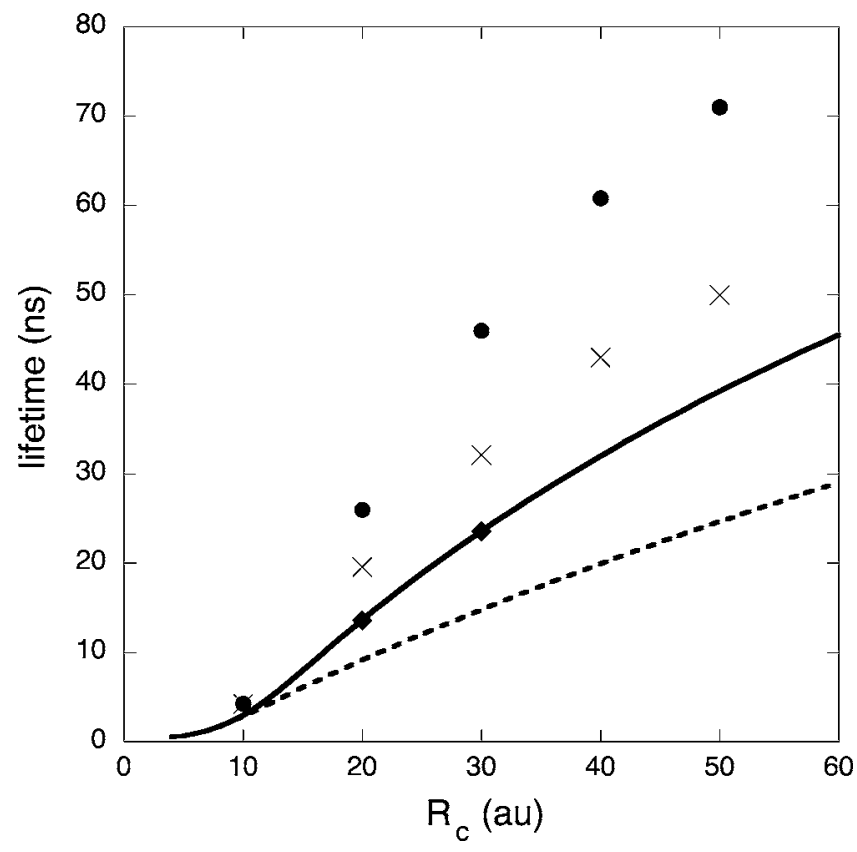

FIG. 4. Curves show lifetimes from Eq. (5) for $m=2$ particle in a pore at two temperatures. Solid line; $\beta=300$, dotted line; $\beta$ $=150$. Diamonds; PIMC simulation with single, $m=2$ particle at $\beta=300$. Filled circles: two-particle PIMC simulation with $\beta=300$. Crosses: two-particle PIMC simulation with $\beta=150$.

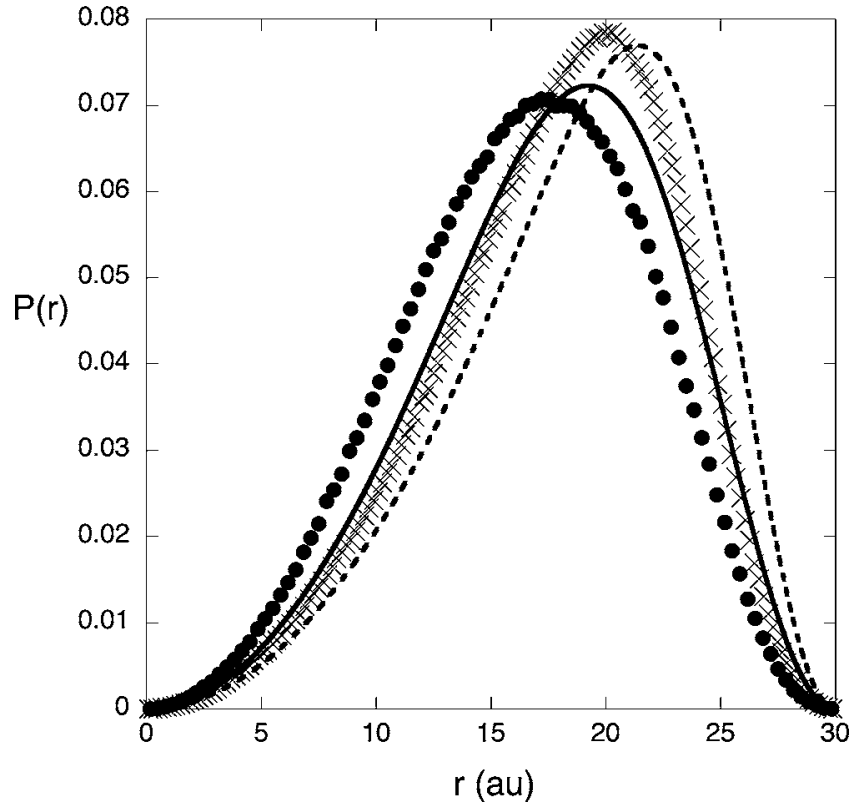

FIG. 5. Curves with symbols are a two-particle prediction of the radial density of $e^{+}, P(r)$, for $R_{c}=30$ a.u.. Filled circles; $\beta=300$. Crosses; $\beta=150$. Curves without symbols represent $\mathrm{P}_{\text {SPIB }}$. Solid line: $\beta=300$; dotted line: $\beta=150$.

of detailed model of the microscopic electronic response to the Ps dipole that neither the PIB nor the current calculation has attempted to provide.

\section{RESULTS FOR MESOPORES}

One must incorporate thermal effects in order to study mesopores ${ }^{8,27}$ for application to, for example, thin films. This is because larger pores have their energy spectrum scaled downward to lower energies. Figure 4 shows the lifetimes from Eq. (3) for a SPIB of Eq. (5) for pores that extend into the mesopore range. This figure shows lifetimes as a function of radius at two different temperatures. A single $m=2$ particle is simulated via PIMC (diamonds) to confirm agreement with Eq. (5). Two-particle PIMC results (circles and crosses in Fig. 4), for $\beta=150$ and 300 are also shown. Figure 5 shows the radial distribution function, $\mathrm{P}(r)$, as compared with $\mathrm{P}_{S P I B}$ for $R=30 \mathrm{a}$.u. data; integration over the outermost $\Delta=3.13$ a.u. of this figure produced the corresponding data points in Fig. 4. As in the case of micropores, the $e^{+}$of Ps avoids the wall as compared with a calculation involving the single particle with $m=2$. This results in a higher value of $\tau$ for the two-particle model. As expected, a higher temperature (lower $\beta$ ) results in a trend of reduced lifetime for both one- and two-particle models. (Eventually, as $R_{c}$ grows, all lifetimes must reach the asymptotic value of $142 \mathrm{~ns}$.) The difference between single particle and two particle results is significantly larger for mesopores than for the micropores discussed in Sec. III. For a pore radius of 40 a.u. the lifetimes for the two models in Fig. 4 differ by a factor of two or more. Similarly, the pore radius corresponding to a lifetime of $40 \mathrm{~ns}$ is about twice as large for the single-particle model as it is for the two-particle simulation. 


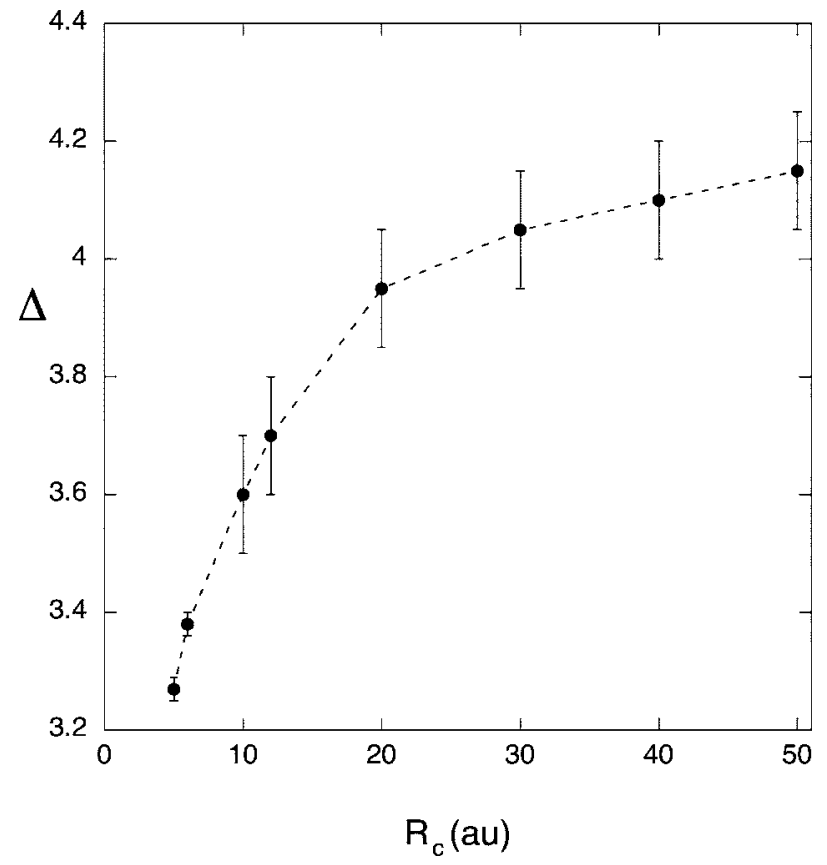

FIG. 6. Effective value of $\Delta$ necessary in order to make lifetime, $\tau$ from two-particle model equal to $\tau_{S P I B}$ in pores at $\beta=300(T$ $=1053 \mathrm{~K})$.

Since the disagreement between the one- and two-particle models can be quite dramatic, it begs the question of how the Tao-Eldrup SPIB model has done so well at predicting lifetimes in both microporous and mesoporous materials. This model, whose single parameter $\Delta$ has been fit for various applications somewhere in the range of 3.0-3.5 a.u. has produced good agreement for pore sizes with other techniques like Brunauer-Emmett-Teller (gas adsorption) or ACAR (measurement of the transverse momentum of the annihilation radiation). How is this possible? It is worthwhile to note that when Eq. (8) holds, which at room temperature is for pore diameters up to roughly 40 a.u., the disagreement between the models is minimal. For $R_{c}=20$, a calculation with $\mathrm{P}=5000$ at $\beta=1060$, corresponding to a temperature of $T=298 \mathrm{~K}$, yields $\tau=21.1 \mathrm{~ns}$, as compared with the SPIB result of $\tau=18.2 \mathrm{~ns}$. The pore radius would be found from the two-particle calculation as 21.2 a. u. which corrects the SPIB estimate by only $5 \%$. It is doubtful that this difference would appear on a fit to experiment.

Calculations of greater complexity, including our current one, surely do not negate the historical utility of the TaoEldrup model. It is hoped, however, that they will guide the inquiry as to how well a certain model might work for a particular application and provide alternatives as appropriate. Based on evidence like those summarized in Tables I and II, one of the categorical assumptions of the Tao-Eldrup potential model (monodisperse, hard-walled pores; no variation of positronic density caused by electronic properties of wall or bulk solid, and so on) may allow it to systematically underestimate a single-particle Ps lifetime in a way that balances the longer lifetime predicted by placing a two-particle Ps in this potential. It has never been suggested that the simplifying assumptions of Tao-Eldrup are rigorously true. TaoEldrup is a simple model that, when its parameters are fit to experiment, works well.

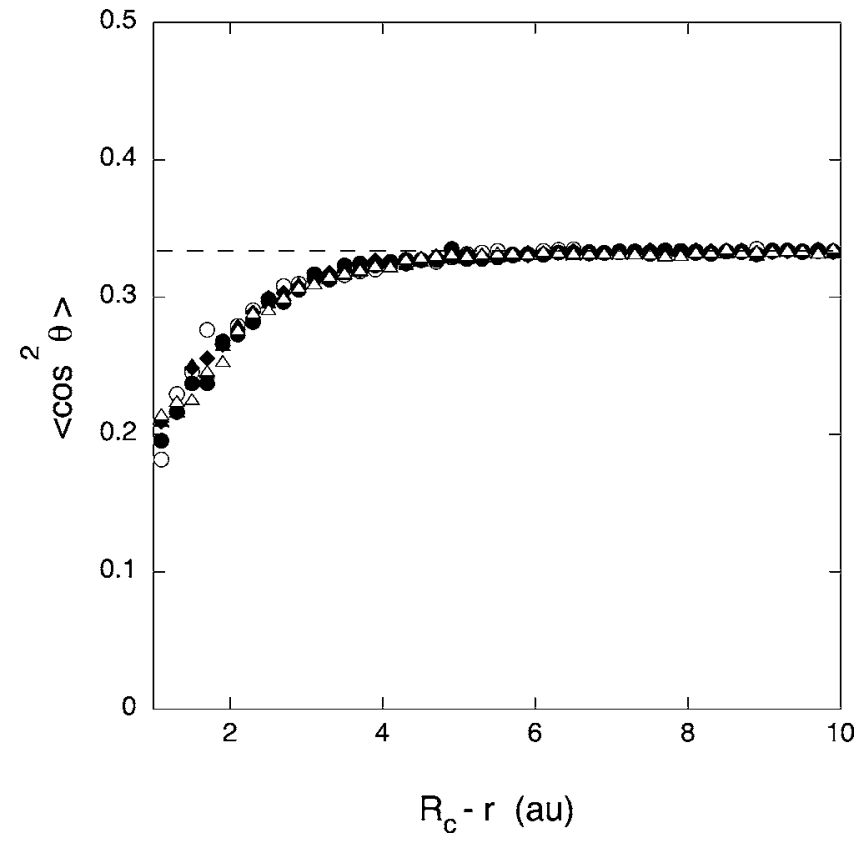

FIG. 7. Expectation value, $\left\langle\cos ^{2} \theta\right\rangle$ as in Fig. 3. For $R_{c}$ $=30$ a.u. Solid circles; $\beta=300$, open circles; $\beta=150$. For $R_{c}$ $=20$ a.u.. Solid diamonds: $\beta=1060$; solid triangles: $\beta=300$; open triangles $\beta=150$. Dashed line indicates the expectation value for an isotropic distribution of orientations: $\left\langle\cos ^{2} \theta\right\rangle=1 / 3$.

Table II, which lists the values of $\Delta\left(R_{c}\right)$ which enforce the agreement between SPIB and two-particle models, is plotted and extended into the mesopore range in Fig. 6. For the case of larger pores, the lifetime was calculated at $T=1053 \mathrm{~K}$, corresponding to $\beta=300$. While this insures ground-state behavior for pores of radius $R_{c}=10 \mathrm{a}$.u. and less, a mixture of excited states contributes to the state of Ps in larger pores. One can see that the effective value of $\Delta$ needed for agreement between the models continues to rise with pore size. One expects $\Delta\left(R_{c}\right)$ to approach an asymptotic value for large $R_{c}$. From Fig. 6, it appears that the asymptote will be somewhat larger than $\Delta=4.1 \mathrm{a} . \mathrm{u}$. for $T=1053 \mathrm{~K}$, and it is unknown whether it has a strong temperature dependence. (Preliminary data at other temperatures suggest that it does not.) This asymptote represents a sizable departure from the range of $\Delta=3.0-3.5$ a.u. used for a range of materials in SPIB models.

Figure 7 shows the orientational order parameter, $\left\langle\cos ^{2} \theta\right\rangle$, as defined in Fig. 3, for mesopores with $R_{c}=20$ and 30. The parameter is plotted as a function of distance from the pore wall. One can see that the shape of the drop-off does not seem to depend on the size of the mesopore. Nor does it depend obviously on the temperature, in the range that we have studied (from room temperature to ten times room temperature). In all cases, the orientational order parameter falls to less than $95 \%$ of its isotropic value when the Ps atom is centered at a distance of $\Delta=3.13 \mathrm{a} . \mathrm{u}$. from the pore wall. Its average value within the entire region of width $\Delta$ is very approximately $3 / 4$ of its isotropic value for all temperatures and both pore sizes studied. The orientation of Ps in a cavity with bulk electrostatic effects included has been reported upon elsewhere. ${ }^{23}$ 


\section{CONCLUSIONS}

A PIMC model of Ps with a composite structure was studied, and compared with a SPIB. In these studies, Ps was trapped in hard spherical pores. The physics is rather different for the two models. The former makes different lifetime predictions, and captures subtle effects that the latter cannot. We have investigated both micropores and moderately-sized mesopores. The contact correlation function, $\kappa$, was found to increase monotonically in pores of radius $12 \mathrm{a} . \mathrm{u}$. and less. Temperature was found to enhance the annihilation rate; and at high temperatures, predictions from the two models could be dramatically different. The $e^{+}, e^{-}$pair tended to turn "side-on" to the pore wall, when its center-of-mass was in close proximity to the wall.

\section{ACKNOWLEDGMENTS}

Many thanks to M. Eldrup, T. Goworek, P. Hastings, Z. Kajcsos, L. Larrimore, M. Muluneh, E. Pollock, H. Saito, and J. Waldman for interesting conversations and/or technical contributions. We are very grateful to T. Hyodo and the organizing committee of ICPA-13, where some of these results were reported. This work was performed under the auspices of the U.S. Department of Energy by the University of California Lawrence Livermore National Laboratory under Contract No. W-7405-Eng-48.
*Author to whom correspondence should be addressed.

${ }^{1}$ S. Dannefaer, D. Kerr, D. Craigen, T. Bretagnon, T. Talierico, and A. Foucaran, J. Appl. Phys. 79, 9110 (1996); D. Bamford, M. Jones, J. Latham, R. J. Hughes, M. A. Alam, J. Stejny, and G. Dlubek, Macromolecules 34, 8156 (2001); Zs. Kajcsos, G. Duplatre, L. Liszkay, K. Lazar, L. Lohonyai, G. Pal-Borbely, H. K. Beyer, P. Caullet, and J. Patarin, Mater. Sci. Forum 363-365, 238 (2001); J. N. Sun, Y. F. Hu, W. E. Frieze, and D. W. Gidley, Radiat. Phys. Chem. 68, 345 (2003); H. Saito and T. Hyodo, Phys. Rev. Lett. 90, 193401 (2003).

${ }^{2}$ Y. C. Jean in Positron Spectroscopy of Solids, edited by A. Dupasquier and A. P. Mills Jr. (IOS Press, Amsterdam, 1995) pp. 563-580.

${ }^{3}$ D. M. Schrader and Y. C. Jean, in Positron and Positronium Chemistry, edited by D. M. Schrader and Y. C. Jean (Elsevier, Amsterdam, 1988), pp. 1-27.

${ }^{4}$ P. Bandzuch, J. Kristiak, O. Sausa, and J. Zrubcova, Phys. Rev. B 61, 8784 (2000).

${ }^{5}$ S. Tao, J. Chem. Phys. 56, 5499 (1972); M. Eldrup, D. Lightbody, and J. N. Sherwood, Chem. Phys. 63, 51 (1981); H. Nakanishi and Y. C. Jean in Positron and Positronium Chemistry, edited by D. M. Schrader and Y. C. Jean (Elsevier, Amsterdam, 1988) Chap. 5; K. Ito, H. Nakanishi, and Y. Ujihira, J. Phys. Chem. B 103, 4555 (1999).

${ }^{6}$ H. Cao, J. -P. Yuan, R. Zhang, C. S. Sundar, Y. C. Jean, R. Suzuki, T. Ohdaira, and B. Nielsen, Appl. Surf. Sci. 149, 116 (1999); T. Goworek, Mater. Sci. Forum 363-365, 227 (2001); F. Faupel, J. Kanzow, K. Gunther-Schade, C. Nagel, P. Sperr, and G. Kogel, ibid. 445-446, 219 (2004); H. -G. Peng, W. E. Frieze, R. Vallery, and D. W. Gidley, Appl. Phys. Lett. 86, 121904 (2005).

${ }^{7}$ G. Consolati, J. Chem. Phys. 117, 7279 (2002).

${ }^{8}$ T. Goworek, B. Jasinska, J. Wawryszczuk, R. Zaleski, and T. Suzuki, Chem. Phys. 280, 295 (2002).

${ }^{9}$ T. Goworek, Chem. Phys. Lett. 366, 184 (2002).

${ }^{10}$ A. Camacho, J. Chem. Phys. 121, 5451 (2004).

${ }^{11}$ In the realm of ab initio quantum-chemical calculations, Tang et $a l$. have recently produced a stable positronium structure in an insulating solid. Z. Tang, M. Hasegawa, Y. Nagai, and M. Saito, Mater. Sci. Forum 445-446, 390 (2004).

${ }^{12}$ P. A. Sterne, L. Larrimore, P. Hastings, and A. L. R. Bug, Radiat.
Phys. Chem. 68, 409 (2003).

${ }^{13}$ P. Hastings, A. L. R. Bug, and P. Sterne, Recent Research Reports of the 13th International Zeolite Conference, Groupe Francais des Zeolithes, Paris, 2001 (unpublished).

${ }^{14}$ B. N. Miller and T. L. Reese, Nucl. Instrum. Methods Phys. Res. B 192, 176 (2002).

${ }^{15}$ H. Schmitz and F. Muller-Plathe, J. Chem. Phys. 112, 1040 (2000).

${ }^{16}$ L. Larrimore, R. N. McFarland, P. A. Sterne, and A. L. R. Bug, J. Chem. Phys. 113, 10642 (2000).

${ }^{17}$ D. M. Ceperley, Rev. Mod. Phys. 67, 279 (1995); D. P. Landau and K. Binder, A Guide to Monte Carlo Simulations in Statistical Physics (Cambridge University Press, Cambridge, 2000) Chap. 8.2.

${ }^{18}$ D. Chandler and P. G. Wolynes, J. Chem. Phys. 74, 4078 (1981).

${ }^{19}$ E. L. Pollock, Comput. Phys. Commun. 52, 49 (1988).

${ }^{20}$ M. H. Muser and B. J. Berne, J. Chem. Phys. 107, 571 (1997).

${ }^{21}$ P. A. Whitlock and M. H. Kalos, J. Chem. Phys. 30, 361 (1979).

${ }^{22}$ J. Cao and B. J. Berne, J. Chem. Phys. 97, 2382 (1992).

${ }^{23}$ A. L. R. Bug, T. Cronin, P. A. Sterne, and Z. Wolfson in the 8th International Workshop on Positron and Positronium Chemistry, Coimbra, Portugal, 2005 [Rad. Phys. Chem. (to be published)].

${ }^{24}$ A. Dupasquier, in Positron Solid-State Physics, edited by W. Brandt and A. Dupasquier (North-Holland, New York, 1983), p. 485; T. Hyodo, Mater. Sci. Forum 363-365, 233 (2001).

${ }^{25}$ T. Goworek, K. Ciesielski, B. Jasinka, and J. Wawryszczuk, Chem. Phys. Lett. 272, 91 (1997).

${ }^{26}$ I. S. Gradshteyn and I. M. Ryzhik, Table of Integrals, Series and Products, 6th ed. (Academic Press, San Diego, 2000), p. 624.

${ }^{27}$ D. W. Gidley, W. E. Frieze, T. L. Dull, A. F. Yee, E. T. Ryan, and H. -M. Ho, Phys. Rev. B 60, R5157 (1999).

${ }^{28}$ W. Brandt, S. Berko, and W. W. Walker, Phys. Rev. 120, 1289 (1960).

${ }^{29}$ A. Michels, J. DeBoer, and A. Bijl, Physica (Amsterdam) 4, 981 (1937).

${ }^{30}$ The centroid of a single, $m=2$ PIMC chain (not shown in Fig. 2) was always seen to be shifted larger radii than the average centroid of the two-particle PIMC model for the same temperature and pore size. This is another illustration of the tendency for the single-particle model to overestimate the positron density near the pore surface. 